\title{
Comparative Study of Distributed Estimation Precision by Average Consensus Weight Models
}

\author{
Martin Kenyeres and Jozef Kenyeres
}

\begin{abstract}
Distributed algorithms for an aggregate function estimation are an important complement of many real-life applications based on wireless sensor networks. Achieving a high precision of an estimation in a shorter time can optimize the overall energy consumption. Therefore, the choice of a proper distributed algorithm is an important part of an application design. In this study, we focus our attention on the average consensus algorithm and evaluate six weight models appropriate for the implementation into real-life applications. Our aim is to find the most suitable model in terms of the estimation precision in various phases of the algorithm. We examine the deviation of the least precise estimate over iterations for a Gaussian, a Uniform and a Bernoulli distribution of the initial states in strongly and weakly connected networks with a randomly generated topology. We examine which model is the most and the least precise in various phases. Based on these findings, we determine the most suitable model for real-life applications.
\end{abstract}

Index Terms-Distributed computing, wireless sensor networks, average consensus algorithm, estimation precision

\section{INTRODUCTION}

\section{A. Estimation techniques in wireless sensor networks}

TN general, the estimation techniques in wireless sensor Inetworks (WSNs) can be classified as centralized or decentralized. The first category requires the presence of the fusion center, whose goal is to gather the information from the other nodes in the network and process it subsequently [1]. This architecture is based on either a massive communication in an extensive geographical area or the implementation of a

Manuscript received September 11, 2017; revised October 28, 2017. Date of publication December 21, 2017

Research described in this paper was financed by the National Sustainability Program under grant LO1401. For the research, infrastructure of the SIX Center was used.

Martin Kenyeres is with Department of Telecommunications, Brno University of Technology, Technicka 12, Brno, Czech Republic. (e-mail kenyeres@phd.feec.vutbr.cz).

Jozef Kenyeres is with Sipwise GmbH, Europaring F15, 2345 Brunn am Gebirge, Austria. (e-mail: jkenyeres@sipwise.com).

Digital Object Identifier (DOI): 10.24138/jcomss.v13i4.405 mechanism for a multi-hop routing (including establishing and maintaining a routing table), which results in a decreased scalability $[1,2]$. This solution is not preferred for real-life applications due to several constraints such as low suitability, low natural robustness, the necessity of the fusion center awareness about the measurement models etc. [3]. Thus, the modern solutions usually utilize the decentralized estimation techniques without the presence of the central fusion center in a network [4]. Their advantages are that the nodes do not have to be aware of network parameters and that it is not necessary to implement any routing protocol. This architecture optimizes the energy consumption (its optimization is a crucial aspect [5]), the natural robustness, the scalability etc. due to a neighbor-to-neighbor communication, on which it is based. Thus, these are the main reasons of why the decentralized estimation techniques are significantly preferred for real-life applications. Decentralized estimation consists of two categories. The first one is based on a sequential transmission of information from one node to another one. The other category is characterized by the diffusion of information into the network [4, 6], which ensures a higher robustness compared with the first category. This is achieved at the cost of a more complicated communication overhead. This other category includes the distributed algorithms [7, 8]. The applications of WSNs based on the presence of the fusion center can also utilize the decentralized estimation techniques. However, this approach results in several constraints such as an increase of transmission power due to the necessity to transmit data to the fusion center, lack of the robustness in a case when the fusion center fails etc [9]. Distributed estimation is a key technology for a wider range of event classification and object tracking in WSNs [10, 11]. A literature review provides many distributed estimation algorithms that find a wide usage in WSNs. In [12], cooperative information aggregation (CIA) schemes used for addressing the distributed estimation problem in WSNs are presented. This approach exploits multi-bit quantizer to quantize the observed signal. Subsequently, the nodes forward only one bit to the fusion center in order to prolong the lifetime of a network. The paper [13] presents an asynchronous distributed estimation technique that is based on a Bayesian model with an unknown hyperparameter. Its principle lays in a distributed computation of MMSE 
(minimum mean square error) estimator of the local arrival rate. In [14], an optimal energy-constrained distributed equivalent unit-energy MSE function are proposed. The authors of [15] propose likelihood consensus method based on iterative consensus algorithms to compute JLF. This function has a key role as it poses the measurements of all the nodes in a network. Distributed estimation techniques get a widespread attention, especially as an application of linear consensus algorithms, which are characterized by minimal communication, computation and synchronization requirements. This fact makes these algorithms suitable for WSNs $[13,16,17,18,19,20,21]$. The roots of these algorithms are in the analysis of the Markov chains and except for the distributed estimation, they find the usage in load balancing, asynchronous solutions for linear systems, cooperative coordination of multi-agent systems etc. [22]. Their principle is based on an interaction of adjacent nodes by means of a diffusion-like process, i.e. the nodes update their local estimates by suitably combining its state with the states collected from the adjacent area [13].

\section{B. Average consensus algorithm}

In this paper, we focus our attention on the average consensus algorithm for an average value estimation. It is a distributed flexible multifunctional consensus algorithm that finds a wide usage in WSNs due to its low computational and energy requirements [23]. Its flexibility is ensured by the possibility to apply various weight models [24]. Each weight model is characterized by a different weight matrix, which affects the aspects such as the convergence rate, the natural robustness, the initial configuration, the required knowledge for the proper functionality etc. The algorithm is based on a mutual exchange of the current inner states between adjacent nodes and updating their inner states according to the collected data from the adjacent area and the value of the inner state from the previous iteration [25]. This procedure is repeated at each iteration until the nodes reach the consensus on the inner states. As mentioned earlier, several weight models have been proposed for the average consensus algorithm. In this paper, we focus our attention on six various models (we assume such weight models that require the information assessable in a distributed manner). We choose the Constant (abbreviated as $\mathrm{CW}$ ), the Maximum Degree (abbreviated as MD), the Metropolis-Hastings (abbreviated as $\mathrm{MH}$ ), the Local Degree (abbreviated as LD), the Best Constant (abbreviated as BC) and the Biphasically configured Metropolis-Hastings weight model (abbreviated as BM). We verify the precision of these weight models, i.e. the deviation of the least precise estimate from the real value of the average in various phases of the algorithm in order to show which one is the most precise and how the precision differs over the algorithm execution.

\section{Motivation}

The overall energy consumption may be significantly optimized by selecting a model that can estimate the average with a high precision in a shorter time. Many of recent papers estimation algorithm and a quasi-optimal energy-constrained distributed estimation algorithm based on a concept of discuss the problem as the energy consumption optimization and put a great emphasis on this aspect [26, 27]. Thus, this motivates us to examine the precision of the chosen weight models in various phases of the algorithm and in different randomly generated networks in order to show which model is the most suitable in terms of the precision in various phases of the algorithm - a higher precision in a shorter time results in a decrease of the number of the transmitted messages, the time necessary for the execution of the estimation process etc. and as the results, it optimizes the estimation process in terms of the energy consumption.

\section{Paper organization}

In the second section, we introduce mathematical tools used to model the average consensus algorithm in WSNs and introduce the weight models of our interest. In the third one, we pay attention to a presentation of the achieved results from our numerical experiments. Furthermore, we examine the highest and the lowest precision of the chosen weight models in various phases of the algorithm and conclude which model is the most suitable for the real-life applications.

\section{Modeling OF Average Consensus}

\section{A. Average consensus in WSNs}

WSNs are usually described as an indirect finite graph $G=(\mathbf{V}, \mathbf{E})$ formed by the sets $\mathbf{V}$ and $\mathbf{E}$ [23]. The set $\mathbf{V}$ consists of all the vertices, which are representatives of the particular nodes. The vertices are identified by the unique identity number $v_{i}$. The size of this set is determined by the number of the nodes in the network (this parameter is labeled as $N$ ). The set $\mathbf{E} \subset \mathbf{V} \mathbf{x V}$ contains all the edges, which represent the direct connection between two nodes $\left(v_{i}, v_{j}\right)$. Sometimes, the label $e_{i j}$ can be found in the literature.

The mutual connectivity among the nodes can be described by the Laplacian matrix $\mathbf{L}$. It is a diagonally symmetric matrix of a square shape defined as [28]:

$$
[L]_{i j}=\left\{\begin{array}{l}
-1, \text { if } e_{i j} \in \mathbf{E} \\
d_{i}, \quad \text { if } i=j \\
0, \text { otherwise, }
\end{array}\right.
$$

Here, $d_{i}$ labels the degree (i.e. the number of the neighbors) corresponding to the node $v_{i}$. Except for the description of the mutual connectivity, the Laplacian matrix provides also additional information about the network topology. If $\lambda_{2}(\mathbf{L})$ (the second smallest eigenvalue of the corresponding Laplacian matrix) is equaled to 0 , then the network is connected [29]. So, only the networks described by the Laplacian matrices with $\lambda_{2}(\mathbf{L})$ not equaled to 0 are connected. In disconnected networks, the nodes estimate the average from the connected subparts and therefore, the algorithm is unable to fulfill its functionality. Thus, our attention is focused only on networks whose $\lambda_{2}(\mathbf{L}) \neq 0$. Furthermore, the knowledge 
about $\lambda_{2}(\mathbf{L})$ and $\lambda_{N}(\mathbf{L})$ is necessary for the optimized variant of the Best Constant weight model.

As previously mentioned, the average consensus algorithm is based on mutual exchanges of the inner states and the asymptotic convergence to the value of an aggregate function. Mathematically, it can be modeled as follows [30]:

$$
\mathbf{x}(k+1)=\mathbf{W} \mathbf{x}(k)
$$

Here, $\mathbf{W}$ is the weight matrix, whose elements are determined by the chosen weight model. The column vector $\mathbf{x}(k) \in \mathbf{R}^{N x I}$ is variant over the iterations and consists of the inner states of all the nodes at each iteration. We assume that the initial iteration is labelled as $k=1$ and so, $\mathbf{x}(1)=\mathbf{x}^{\text {in }}$. The elements of the weight matrix affect the convergence rate of the algorithm as well as whether the algorithm converges or diverges. The convergence conditions, which the weight matrix $\mathbf{W}$ has to hold, are discussed in [31].

Since the average consensus algorithm is an iterative algorithm asymptotically converging to the average, the following statement is valid [14]:

$$
\lim _{k \rightarrow \infty} \mathbf{x}(k)=\lim _{k \rightarrow \infty} \mathbf{W}^{k-1} \mathbf{x}(1)=\frac{\mathbf{1 1}^{\mathrm{T}}}{N} \mathbf{x}(1)
$$

The vector $\mathbf{1}$ has a column shape and all its elements are equal to 1 .

\section{B. Examined weight models of average consensus algorithm}

In the following section, we introduce the weight models chosen for our examination. The first one is the Constant weight model defined as follows [31]:

$$
\left[W^{\mathrm{Cw}}\right]_{i j}=\left\{\begin{array}{cc}
\varepsilon, & \text { if }\left(v_{i}, v_{j}\right) \in \mathbf{E} \\
1-d_{i} \varepsilon, & \text { if } i=j \\
0, & \text { otherwise }
\end{array}\right.
$$

This model is characterized by the mixing parameter $\varepsilon$, whose value determines the convergence rate of the algorithm and whether the algorithm convergences or diverges [32]. According to [31], its value has to be chosen from the following interval so that the convergence is achieved:

$$
0<\varepsilon \leq \frac{1}{d_{\max }}
$$

Here, $d_{\max }$ is the number of the neighbors of the bestconnected node in a network. Due to the limited size of the paper, we choose the value of $\varepsilon$ equaled to $0.5 * 1 / d_{\max }$ as a representative of this weight model. As analyzed in [33], higher values of $\varepsilon$ ensure a higher convergence rate of the algorithm. On the other hand, the algorithm is less robust to several types of failures. Therefore, it makes a sense to use a more conservative initial setup, especially in systems with a high error rate. We choose $0.5^{*} 1 / d_{\max }$ as a compromise between the convergence rate and the failure tolerance.

The next model of the interest is the Maximum Degree weight model. Based on the Constant weight model, the value of $\varepsilon$ is set to the maximal possible value ensuring the convergence and therefore, to $1 / d_{\max }$. Thus its weight model is defined as follows [34]:

$$
\left[W^{\mathrm{MD}}\right]_{i j}=\left\{\begin{array}{cc}
1 / d_{\text {max }}, & \text { if }\left(v_{i}, v_{j}\right) \in \mathbf{E} \\
1-d_{i} / d_{\max }, & \text { if } i=j \\
0, & 0
\end{array}\right.
$$

Two other models are the Metropolis-Hastings and the Local degree weight model, which both do not have uniform weights. These two models are very appropriate for real-life applications because they need only locally available information for the initial configuration. Their weight matrices can be defined as follows [35, 36]:

$$
\begin{gathered}
{\left[W^{\mathrm{MH}}\right]_{i j}=\left\{\begin{array}{cc}
1 /\left(1+\max \left\{d_{i}, d_{j}\right\}\right), & \text { if }\left(v_{i}, v_{j}\right) \in \mathbf{E} \\
1-\sum_{k=1, k \neq i}^{N}\left[W^{\mathrm{MH}}\right]_{i k}, & \text { if } i=j \\
0, & \text { otherwise, }
\end{array}\right.} \\
{\left[W^{\mathrm{LD}}\right]_{i j}=\left\{\begin{array}{cc}
1 / \max \left\{d_{i}, d_{j}\right\}, & \text { if }\left(v_{i}, v_{j}\right) \in \mathbf{E} \\
1-\sum_{k=1, k \neq i}^{N}\left[W^{\mathrm{LD}}\right]_{i k}, & \text { if } i=j \\
0, & \text { otherwise, }
\end{array}\right.}
\end{gathered}
$$

The next model is the Best Constant weight model, which requires the knowledge about the largest and the second smallest eigenvalue of the corresponding Laplacian matrix and therefore, its optimized variant posses a significant challenge to be implemented. Nevertheless, in the experimental part, we assume its optimized variant defined as [35]:

$$
\left[W^{\mathrm{BC}}\right]_{i j}=\left\{\begin{array}{cc}
2 /\left(\lambda_{2}(\mathbf{L})+\lambda_{N}(\mathbf{L})\right), & \text { if }\left(v_{i}, v_{j}\right) \in \mathbf{E} \\
1-2 d_{i} /\left(\lambda_{2}(\mathbf{L})+\lambda_{N}(\mathbf{L})\right), & \text { if } i=j \\
0, & \text { otherwise }
\end{array}\right.
$$

Here, $\lambda_{2}(\mathbf{L})$ and $\lambda_{N}(\mathbf{L})$ are the second smallest and the largest eigenvalue of the corresponding Laplacian matrix.

The last examined model is the Biphasically configured Metropolis-Hastings weight model, which is derived from the Metropolis-Hastings weight model [36]. Its principle is based on a decrease of the weights allocated to the inner states by increasing the weights of the nodes from the adjacent area. As adduced in [36], the convergence rate is affected by the position of the initial identity numbers. Therefore, we repeat each experiment 100 times and choose the average as a representative of this set.

\section{EXPERIMENTAL PART AND DISCUSSION}

In the experimental part, we examine how the precision of the weight models differs in various phases of the algorithm execution using numerical experiments executed in Matlab. We examine the value of the relative deviation of the least precise estimate from the real value of the average at ten iterations $\left\{100^{\text {th }}\right.$ it., $200^{\text {th }}$ it., $300^{\text {th }}$ it., $\ldots .1000^{\text {th }}$ it. $\}$. We demonstrate our intention on ten strongly and ten weakly connected networks with a topology randomly generated as described in [16] (see Fig. 1 and Fig. 2 for representatives). 


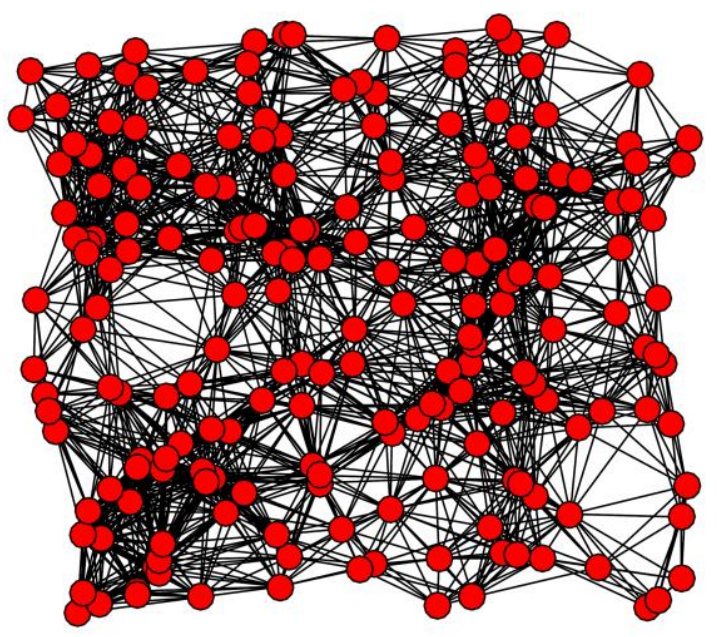

Fig. 1. Representative of strongly connected networks

Within our analysis, we assume that the initial states are allocated randomly generated values of a Gaussian, a Uniform and a Bernoulli distribution with the following parameters:

- Gaussian distribution: $\mu=100, \delta=1$

- Uniform distribution: $a=0, b=200$

- Bernoulli distribution: the initial state is 1 with the probability equaled to 0,5 , the initial state is 0 with the probability equaled to 0,5 .

Here, $\mu$ represents the value of the mean and $\delta$ is the standard deviation. The parameters $a$ and $b$ are the lower and the upper bound, respectively.

\section{A. Examination of most and least precise weight models}

In the first part of the experimental section, we focus on figuring out which model is the most and the least precise at the chosen iterations for each distribution of the initial states. The results containing the extremes for each phase (i.e. the information about which weight model has the lowest and the highest estimation precision at an adduced iteration) are shown in Table 2 in the Appendix (for each distribution in the strongly connected networks). The columns B and $\mathrm{W}$ contain the information about the weight models with the highest or respectively the lowest precision for the examined weight models in the corresponding phase and network (i.e. the number of the networks in which they achieve the extreme). In the following part, we analyze the achieved results depicted in Table 2. At first, let us focus on the precision in the strongly connected networks. The first part is focused on an examination of the precision of the weight models with the initial states having the Gaussian distribution. Here, CW reaches the lowest precision in all ten networks and in each phase of the algorithm. Regarding the highest precision, BC achieves the highest precision in 47 cases (one case means the highest or the lowest precision in one network and in one phase) and BM in 53 cases. We can see that BM is the most precise in earlier phases of the algorithm, then, these two models achieve the highest precision in the same number of networks in five phases and in later phases, $\mathrm{BC}$ is the most

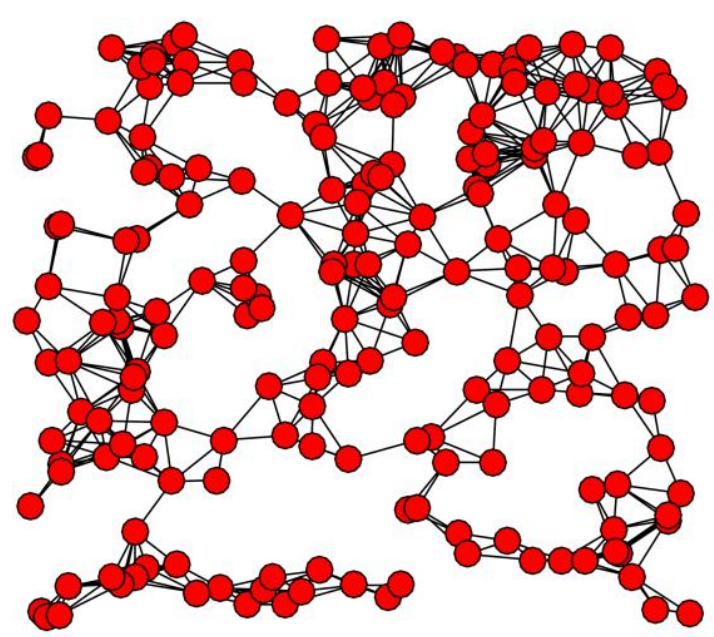

Fig. 2. Representative of weakly connected networks

precise in more networks than BM. In the scenario when the nodes are initiated with randomly generated inner states of the Uniform distribution, we can see that $\mathrm{CW}$ is again the least precise in all networks and in all the phases. As for the weight models with the highest precision, the character of BC (46 cases) and BM (51 cases) is similar to the previous analysis, i.e. $\mathrm{BM}$ is the most precise in earlier phases in more networks than $\mathrm{BC}$, then, both of them achieve the highest precision equally in five networks and in later phases, $\mathrm{BC}$ is the most precise in more networks than BM. In contrast to the previous analysis, also LD achieves the highest precision - it is observed in three networks in earlier phases of the algorithm. Regarding the Bernoulli distribution, here, we can see that $\mathrm{CW}$ is the least precise in 98 cases. In two other cases, it is BC (although it achieves a very high precision in previous two experiments), which reaches the lowest precisions - it happens in earlier phases of the algorithm. Paradoxically, this weight model also achieves the highest precision in four networks when the Bernoulli distribution is used - now, this can be seen in later phases. Another model that achieves the highest precision at least in one case is BM, which achieves the best result in 96 cases.

In the overall evaluation (see Fig. 3 and Fig. 4), BM achieves the highest precision in the most number of the cases $(67 \%)$. It is the most precise model especially in earlier phases of the algorithm. The second best is BC (32\%) even though it reaches the worst precision in two networks in earlier phases. Nevertheless, this weight model achieves a very high precision especially in later phases, where it achieves the highest precision in more networks than BM except for the cases when the initial states are of the Bernoulli distribution. Furthermore, we can see that also LD (in $1 \%$ of the cases) has the highest precision in three networks in earlier phases. Regarding the least precise weight models, CW (in 99\%) achieves the smallest precision in all the networks and in all the phases except for two cases when BC $(<1 \%)$ achieves less precision than this model.

In the next part, we analyze the precision in the weakly 


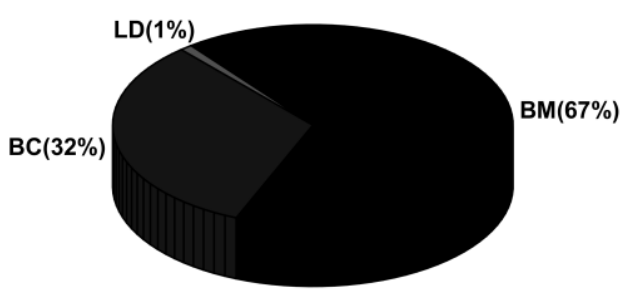

Fig. 3. Weight models with highest precision in strongly connected networks (percentage ratio)

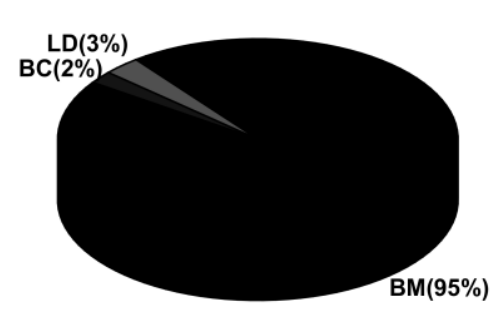

Fig. 5. Weight models with highest precision in weakly connected networks (percentage ratio)

connected networks (Table 2). At first, we again focus on the scenario when the initial states are of the Gaussian distribution. Here, $\mathrm{CW}$ reaches the lowest precision in all cases just like in the strongly connected networks. The highest precision is reached by BM in all the cases except for six when $\mathrm{BC}$ is more precise. However, in contrast to the experiments executed in the strongly connected networks, BC does not achieve the highest precision in later phases of the algorithm. Regarding the experiment with the Uniform distribution, the lowest precision is achieved by $\mathrm{CW}$ in 70 cases and $\mathrm{BC}$ in 30 cases. BC achieves the lowest precision more frequently in earlier phases of the algorithm. Regarding the highest precision, BM achieves the best results in 91 cases and LD in 9 cases (the distribution is almost uniform within all the phases of the algorithm). For the Bernoulli distribution, $\mathrm{CW}$ achieves the lowest precision in 56 cases and $\mathrm{BC}$ in 44 cases. $\mathrm{BC}$ achieves the lowest precision in the most networks only at the $100^{\text {th }}$ iteration, then at the $200^{\text {th }}$ iteration, both weight models have the lowest precision in the same number of the networks and from the $300^{\text {th }}$ iteration, $\mathrm{CW}$ is the least precise in more networks than $\mathrm{BC}$. BM achieves the highest precision in all 100 cases.

Overall (see Fig. 5 and Fig. 6), we can see that BM achieves the highest precision in $95 \%$ of the cases and so, is a significantly more precise than the other examined weight models in weakly connected networks. LD is the second most precise weight model and achieves the highest precision in 3 $\%$ of the cases. The third most precise model is BC, which is the most precise in 3 cases in earlier phases of the algorithm (2 $\%)$. Regarding the least precise weight models, the worst one is $\mathrm{CW}$, which achieves the lowest precision in $75 \%$ of the cases. The other model that has the lowest precision at least in one case is BC (in $25 \%$ ).

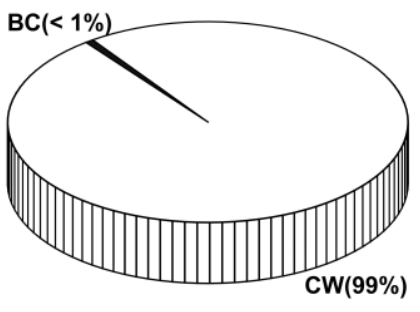

Fig. 4. Weight models with lowest precision in strongly connected networks (percentage ratio)

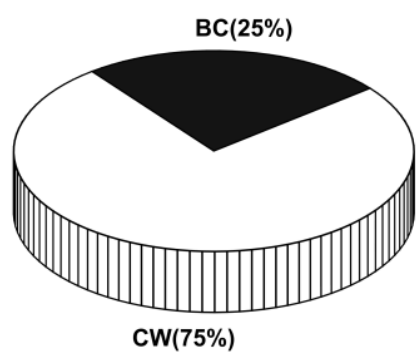

Fig. 6. Weight models with lowest precision in weakly connected networks (percentage ratio)

The complete comparison (the extremes in the strongly and the weakly connected networks are shown together in one graph depicted in Fig. 7).

\section{B. Estimation precision by examined weight models}

In the next section, our attention is turned to the average values of the estimation precision by the examined weight models. We depict the relative average deviation calculated from the least precise estimates in all ten networks for both the strongly and the weakly connected ones, the maximal and the minimal deviation of the least precise estimate within ten networks for each iteration and for each distribution of the initial values.

Initially, we focus on an analysis of the average relative deviations of the least precise estimates in each examined phase of the algorithm. We depict the values calculated as the average from the relative deviations in all ten networks separately for each phase and each distribution of the initial values. The results are shown in Fig. 8 - Fig. 13 and in Table 1. The average deviation in the strongly connected networks initiated with states of the Gaussian distribution decreases as the number of the iterations grows for each examined model. For the Gaussian distribution, the least precise model is CW, whose deviations are from the interval $<8,3 \mathrm{E}-01 \%, 1,1 \mathrm{E}-$ $02 \%>$. MD is the second lowest precise weight model and its average deviations take the values from $<3,8 \mathrm{E}-01 \%, 4,1 \mathrm{E}-$ $04 \%>$. The third model in terms of the average precision is BC with $\langle 2,3 \mathrm{E}-01,1,2 \mathrm{E}-06\rangle$. The fourth and the fifth models are $\mathrm{MH}$ with $\langle 9,6 \mathrm{E}-02 \%, 5,7 \mathrm{E}-09 \%\rangle$, and LD with $\langle 8,2 \mathrm{E}-02 \%$, $1,2 \mathrm{E}-09 \%\rangle$. The most precise at all the iterations is $\mathrm{BM}$ $<7,3 \mathrm{E}-02 \%, 1,4 \mathrm{E}-10\rangle$.

The performance of the weight models regarding the 

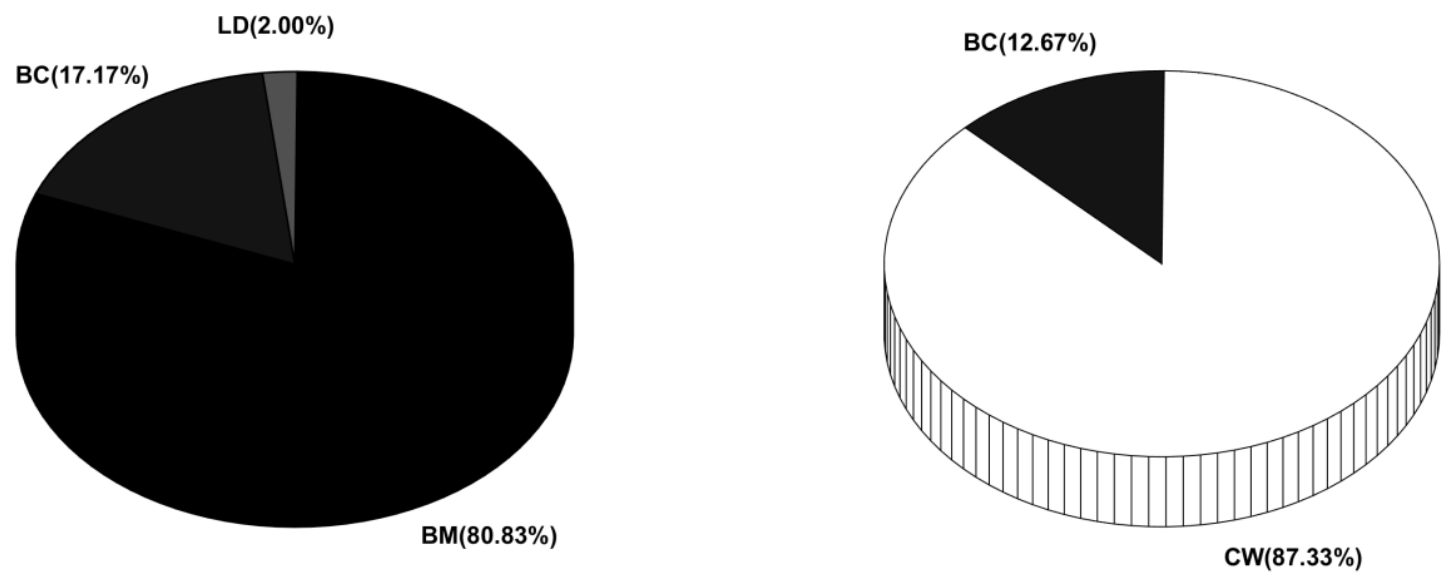

Fig. 7. Overall evaluation of examined weight models - weight models with highest (left) and lowest (right) precision

estimation precision for the Uniform distribution and the Bernoulli distribution does not vary from the previous scenario, i.e. $\mathrm{CW}$ has the smallest average precision in each phase, MD is the second least precise etc.. However, the values of the deviations are significantly higher for the Uniform distribution of the initial values compared with the Gaussian one. CW takes the values from $<8,5 \mathrm{E}+01 \%$, $1,8 \mathrm{E}+00 \%\rangle$, MD from $\langle 4,5 \mathrm{E}+01 \%, 6,8 \mathrm{E}-02 \%\rangle, \quad \mathrm{BC}$ $\langle 3,1 \mathrm{E}+01 \%, 3,0 \mathrm{E}-04 \%\rangle, \mathrm{MH}<10.71 \%, 7.900937 \mathrm{e}-07 \%\rangle$, $\mathrm{LD}\langle 9,0 \mathrm{E}+00 \%, 1,7 \mathrm{E}-07 \%\rangle$, and $\mathrm{BM}<7,8 \mathrm{E}+00 \%$, 1,7E$08 \%>$. For the Bernoulli distribution, the deviation of $\mathrm{CW}$ takes the values from $\langle 1,4 \mathrm{E}+02 \%, 6,4 \mathrm{E}-01 \%\rangle$, MD $\langle 6,1 \mathrm{E}+01 \%, 1,5 \mathrm{E}-02 \%\rangle, \mathrm{BC}\langle 5,4 \mathrm{E}+01 \%, 5,4 \mathrm{E}-04 \%\rangle, \mathrm{MH}$ $\langle 1,3 \mathrm{E}+01 \%, 3,1 \mathrm{E}-07 \%\rangle$, LD $\langle 1,0 \mathrm{E}+01 \%, 7,1 \mathrm{E}-08 \%\rangle$ and $\mathrm{BM}\langle 3,7 \mathrm{E}-02 \%, 4,1 \mathrm{E}-11 \%\rangle$. The precision is smaller compared with the Gaussian distribution except for BM, which achieves a higher precision in all the phases for the Bernoulli distribution in $(\langle 3,7 \mathrm{E}-02 \%, 4,1 \mathrm{E}-11 \%\rangle)$. A comparison of the results for the Uniform and the Bernoulli distribution shows that $\mathrm{MD}, \mathrm{MH}$, and $\mathrm{LD}$ achieves a higher precision for the Bernoulli distribution except for the 100th iteration. $\mathrm{CW}$ also achieves a higher precision for the Bernoulli distribution excluding the $100^{\text {th }}$, the $200^{\text {th }}$, and the $300^{\text {th }}$ iteration. $\mathrm{BC}$ is extremely imprecise for the Bernoulli distribution and so, the precision is lower at each iteration compared with the Uniform distribution. Unlike BC, BM achieves a very high precision, i.e. it is more precise at each iteration compared with the Uniform distribution.

In the following paragraphs, we turn our attention to the results achieved in the weakly connected networks. Like in the previous analysis, the deviation of the least precise estimates decreases as the number of the iteration grows regardless of the underlying weight model and the distribution of the initial states. For the Gaussian distribution, $\mathrm{CW}$ has the lowest average precision at each iteration, taking values from $<3,7 \mathrm{E}+00 \%, 3,7 \mathrm{E}-01 \%\rangle$. The second lowest precise is $\mathrm{MD}$ $\langle 2,1 \mathrm{E}+00 \%, \quad 1,6 \mathrm{E}-01 \%\rangle, \quad \mathrm{BC}$ is the third imprecise $<1,5 \mathrm{E}+00 \%, 6,5 \mathrm{E}-02 \%\rangle$, then, $\mathrm{MH}<9,2 \mathrm{E}-01 \%, 4,3 \mathrm{E}-0 \% 2\rangle$,
$<4,2 \mathrm{E}+01 \%, 7,2 \mathrm{E}-01 \%\rangle$. The models achieve a significantly lower precision compared with the scenario when the Gaussian distribution is used like in the strongly connected networks. Regarding the Bernoulli distribution, we can see again that the order of the weight models sorted according to the estimation precision changes like for the Uniform distribution. However, in this case, BC is the least precise at the $100^{\text {th }}-$ the $700^{\text {th }}$ iteration. For the rest of the iterations, this weight model is the second least precise. Its average deviations of the least precise estimates are from $<4,8 \mathrm{E}+02 \%$, $4,3 \mathrm{E}+01 \%>$. CW is the second least precise at the 100th - the 700th iteration and the most imprecise at the 800th - the 1000 th iteration. Then, the next models are MD $<2,1 \mathrm{E}+02 \%$, $2,2 \mathrm{E}+01 \%\rangle, \quad \mathrm{MH}\langle 1,3 \mathrm{E}+02 \%, \quad 5,5 \mathrm{E}+00 \%\rangle$ and $\mathrm{LD}$ $\langle 1,1 \mathrm{E}+02 \%, 3,6 \mathrm{E}+00 \%\rangle$. BM reaches the highest average precision $\langle 3,9 \mathrm{E}-01 \%, 1,2 \mathrm{E}-02 \%\rangle$. Compared with the Gaussian distribution of the initial values, the precision for the Bernoulli distribution is lower for each weight models at each iteration except for BM, whose precision is higher at each iteration. The same character (for both BM and the rest weight models) is observed also in a comparison with the Uniform distribution.

A comparison of the achieved precisions in the strongly and the weakly connected networks shows that the algorithm is more precise in the strongly connected networks at the examined iterations regardless of the underlying weight model. This difference is more significantly observable at later iterations. The deviation of the least precise estimates has a declining character in both types of the networks, however, in the strongly connected ones, the decrease is more intensive.

Consequently, we focus our attention on the maximal and the minimal deviation, i.e. we depict two values - the relative deviation in the networks where it achieves the smallest and the largest value, respectively (for each iteration and for each distribution of the initial values separately). From the results (see Fig. 14 - Fig. 19), we can see that in the strongly connected networks, the weight models are sorted in the descending order according to the maximal deviation as 
follows: $\mathrm{CW}, \mathrm{MD}, \mathrm{BC}, \mathrm{MH}, \mathrm{LD}$, and $\mathrm{BM}$. This order is the same for all the examined iterations and the used distributions of the initial values - the same as for the average deviations. $\mathrm{CW}$ achieves also the worst result in terms of the minimal deviation of the least precise estimates at all the examined iterations and for all the distributions of the initial values. For the Gaussian distribution, MD has the second largest minimal deviations at nine iterations and the fourth largest one at one iteration.

For the Uniform distribution, it achieves the second largest minimal deviations at nine iterations and the third highest one at one iteration. In the scenario when the Bernoulli distribution is used, MD has the second largest minimal deviation at all ten iterations for the Bernoulli distribution. Furthermore, this weight model has the second largest minimal deviation at one iteration for the Gaussian and the Uniform distribution.

Regarding LD: for the Gaussian distribution, its largest deviation is the fourth largest at eight iteration, the fifth largest (or the second smallest) at two iterations, and the third largest at one iteration. For the Uniform distribution, it has the fourth largest minimal deviation at seven iterations, the fifth largest one at two iterations, and the smallest one at one iteration. For the Bernoulli distribution, it has the fourth largest minimal deviation at each iteration. $\mathrm{BC}$ has the smallest minimal deviation at the most number of the iterations (eight iterations for the Gaussian distribution, nine iterations for the Uniform

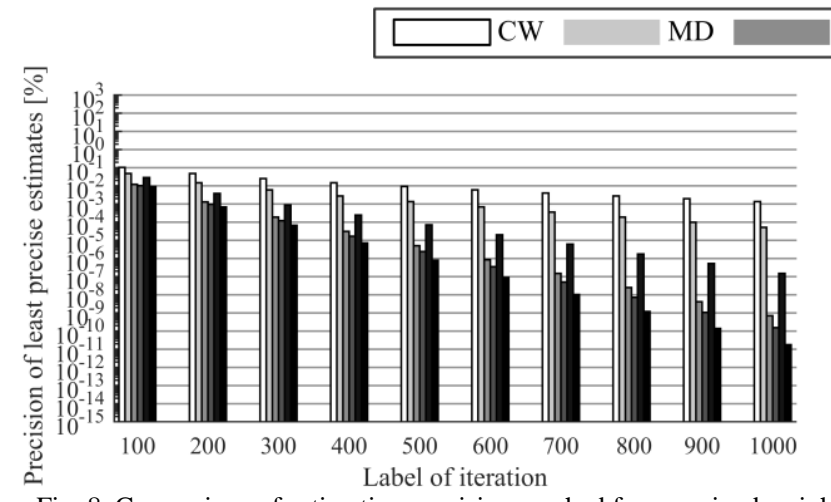

Fig. 8. Comparison of estimation precision reached for examined weight models in strongly connected networks with Gaussian distribution of initial values in various phases of algorithm

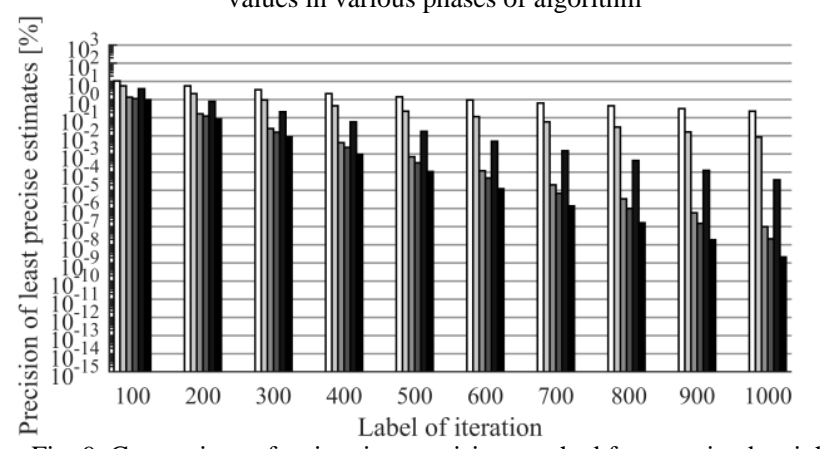

Fig. 9. Comparison of estimation precision reached for examined weight models in strongly connected networks with Uniform distribution of initial values in various phases of algorithm

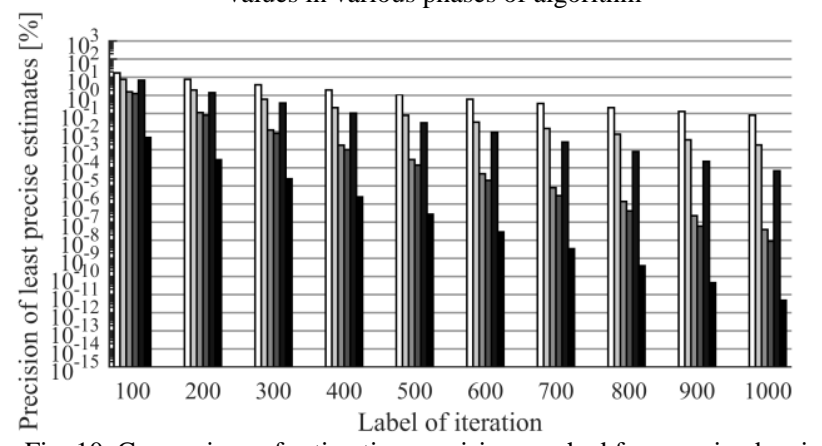

Fig. 10. Comparison of estimation precision reached for examined weight models in strongly connected networks with Bernoulli distribution of initial values in various phases of algorithm

examined iterations. $\mathrm{MH}$ has the third largest minimal deviation at nine iterations for the Gaussian distribution, at nine iterations for the Uniform distribution, and at ten

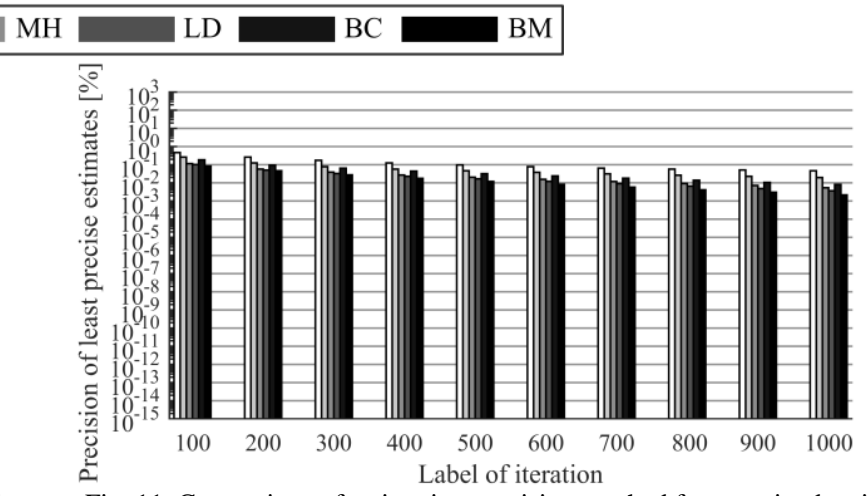

Fig. 11. Comparison of estimation precision reached for examined weight models in weakly connected networks with Gaussian distribution of initial values in various phases of algorithm

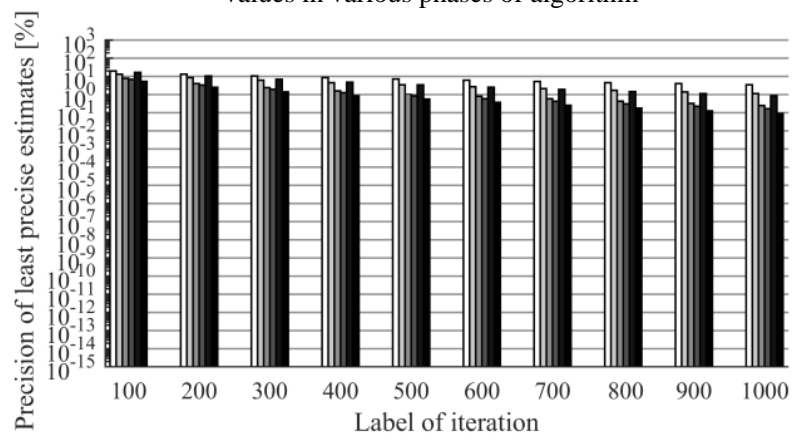

Fig. 12. Comparison of estimation precision reached for examined weight models in weakly connected networks with Uniform distribution of initial values in various phases of algorithm

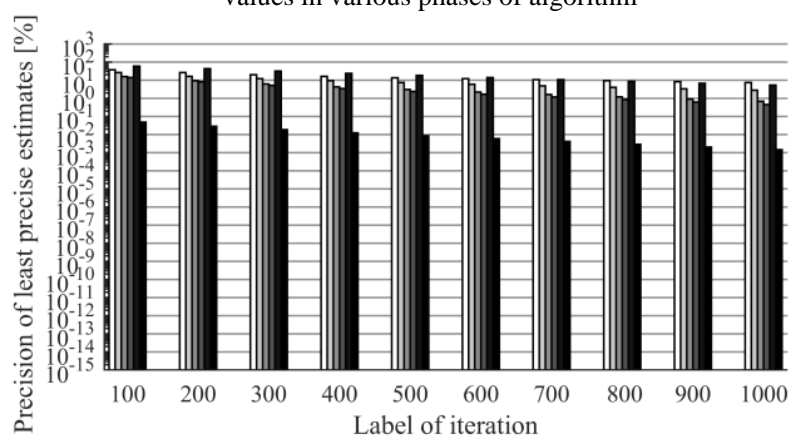

Fig. 13. Comparison of estimation precision reached for examined weight models in weakly connected networks with Bernoulli distribution of initial values in various phases of algorithm distribution). At one iteration for the Gaussian distribution, it has the fourth and fifth largest minimal deviation. Except these, the minimal deviation of $\mathrm{BC}$ achieves the fourth largest 
value at one iteration for the Uniform distribution. For the Bernoulli distribution, its minimal deviation is the fifth largest at each iteration. BM has the fifth largest deviation at seven iterations, the smallest one at two iterations, and the fourth largest one at one iteration for the Gaussian distribution. For the Uniform distribution, the fifth largest deviation is achieved at eight iterations and the fourth largest one at two iterations. For the Bernoulli distribution, BM has the smallest minimal deviation at all ten iterations.

In the weakly connected networks, $\mathrm{CW}$ has the largest maximal deviation at each iteration for the Gaussian and the Uniform distribution and the second largest one at each iteration for the Bernoulli distribution. MD achieves the second largest maximal deviation at seven iterations and the third largest one at three iterations for the Gaussian distribution. For the Uniform distribution, this weight has the second largest maximal deviation at nine iterations and the third largest one at one iteration. For the Bernoulli distribution, MD has the third maximal deviation at each iterations. $\mathrm{MH}$ has the fourth largest maximal deviation at each iteration for each distribution. LD achieves the fifth largest maximal deviation at eight iterations and the smallest one at two iterations for the Gaussian distribution. For the Uniform and the Bernoulli distribution, it has the fifth largest maximal deviation at ten iterations. BC achieves the second largest maximal deviation at three iterations and the third largest one at seven iterations, both for the Gaussian distribution. For the Uniform distribution, this weight model has the second largest deviation at one iteration and the third largest deviation at nine iterations. For the Bernoulli distribution, it achieves the largest maximal deviation at each iterations. BM has the fifth largest maximal deviation at two iterations and the smallest one at eight iterations. For the Uniform and the Bernoulli distribution, this weight has the

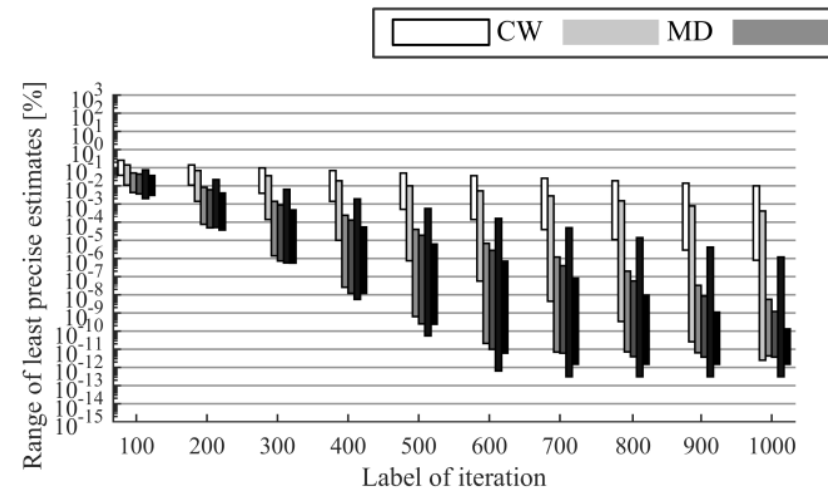

Fig. 14. Comparison of ranges of estimation precision reached for examined weight models in strongly connected networks with Gaussian distribution of initial values in various phases of algorithm

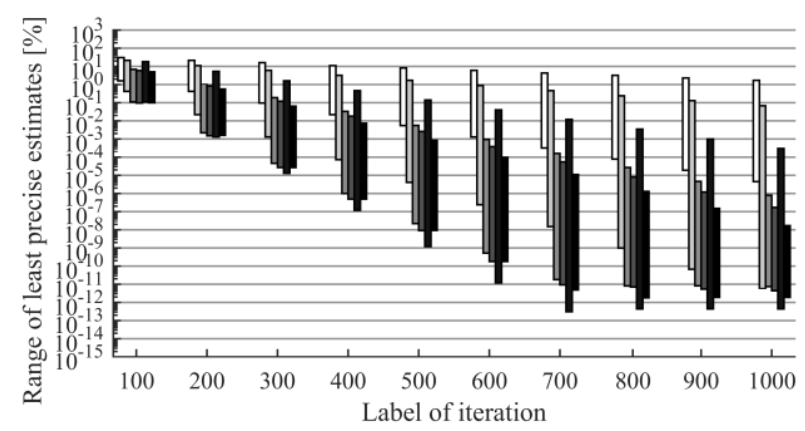

Fig. 15. Comparison of ranges of estimation precision reached for examined weight models in strongly connected networks with Uniform distribution of initial values in various phases of algorithm

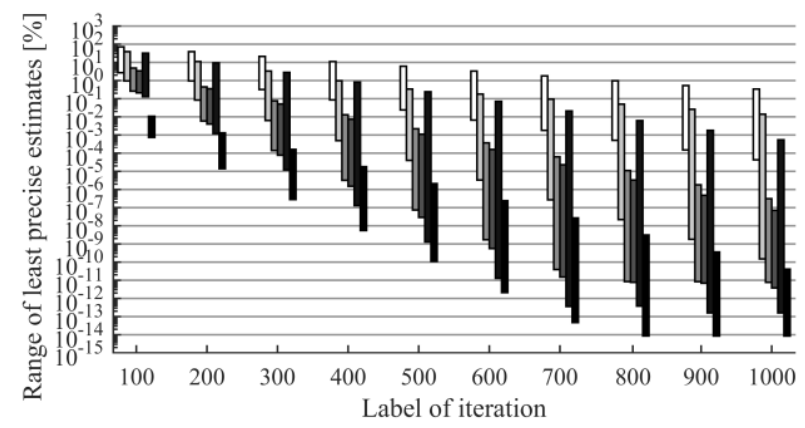

Fig. 16. Comparison of ranges of estimation precision reached for examined weight models in strongly connected networks with Bernoulli distribution of initial values in various phases of algorithm

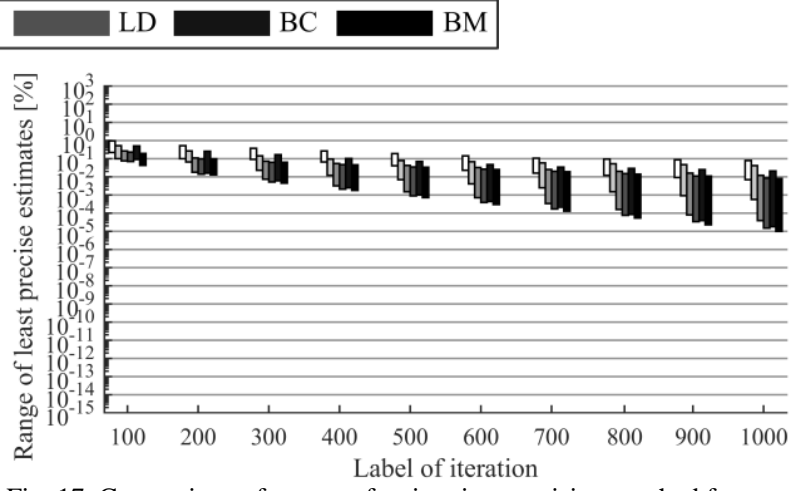

Fig. 17. Comparison of ranges of estimation precision reached for examined weight models in weakly connected networks with Gaussian distribution of initial values in various phases of algorithm

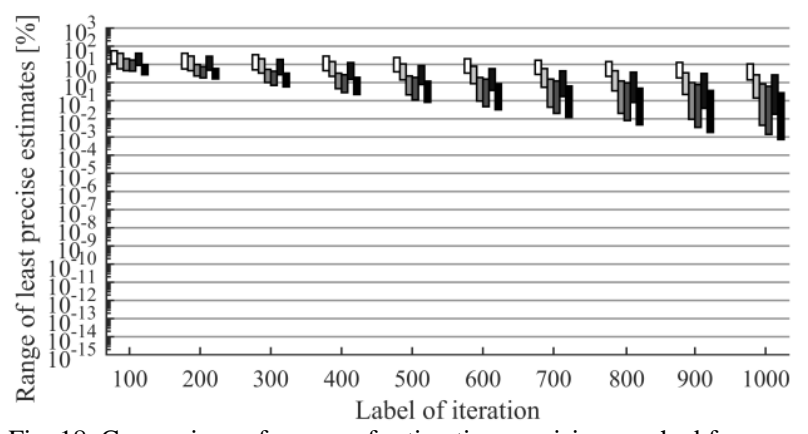

Fig. 18. Comparison of ranges of estimation precision reached for examined weight models in weakly connected networks with Uniform distribution of initial values in various phases of algorithm

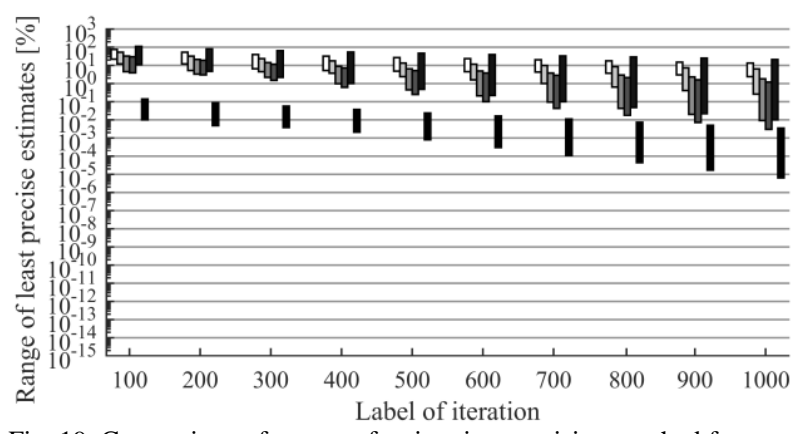

Fig. 19. Comparison of ranges of estimation precision reached for examined weight models in weakly connected networks with Bernoulli distribution of initial values in various phases of algorithm 
smallest deviation at each iteration.

Overall for the Gaussian distribution, the maximal deviation is the smallest for each iteration and weight model except for $\mathrm{BM}$, which achieves the smallest values at each iteration for the Bernoulli distribution. A comparison of the Uniform and the Bernoulli distribution shows that $\mathrm{CW}$ achieves a smaller maximal deviation only at the $100^{\text {th }}-$ the $300^{\text {th }}$ iteration for the Uniform distribution, MD only at the $100^{\text {th }}$, MH achieves better results at each iteration for the Bernoulli distribution, just like LD. Vice versa, BC has a smaller maximal deviation at each iteration for Uniform distribution and BM, as mentioned above, is the best in this aspect for the Bernoulli distribution. Regarding the smallest minimal deviation: the character of the results is the same as in the previous analysis, i.e. for the Gaussian distribution, the maximal deviation is the smallest for each iteration and weight model except for BM, which achieves the best results for the Bernoulli distribution. The smallest minimal deviations for the Uniform distribution are smaller than for the Bernoulli distribution except for LD at the $1000^{\text {th }}$ iteration, $\mathrm{BC}$ at the $800^{\text {th }}-$ the $1000^{\text {th }}$ iteration and for BM at each iteration. So, a comparison between the Uniform and the Gaussian distribution shows that the largest maximal deviations reach smaller values for the Bernoulli distribution, while, the smallest ones for the Uniform one.

Regarding the minimal deviation in the weakly connected networks, CW reaches the largest minimal deviation at each iteration and for each distribution. MD reaches the second largest minimal deviation at each iteration for the Gaussian and the Bernoulli distribution. For the Uniform distribution, the second largest minimal deviation is observed at eight iterations and the third largest one at two iterations. MH has the third largest minimal deviation at nine iterations and the fourth one at one iteration for the Gaussian distribution. For the Uniform and the Bernoulli distribution, it has the fourth largest minimal deviation at each iteration. BC has the third largest minimal deviation at one iteration and the fourth largest one at nine iterations for the Gaussian distribution. For the Uniform distribution, BC achieves the second largest minimal deviation at two iterations and the third largest one at eight iterations. For the Bernoulli distribution, this weight model has the third largest minimal deviation at each iteration. $\mathrm{BM}$ has the largest minimal deviation at each iteration and for each distribution of the initial values.

Overall for the Gaussian distribution, the maximal deviation has the same character as for in the strongly connected networks. Regarding the Uniform distribution, all the models achieve a smaller maximal deviation at each iteration compared with the Bernoulli distribution except for BM. The smallest minimal deviations take the lowest value for the Gaussian distribution for each iteration and each weight model except for BM, whose smallest deviations take the smallest values at the $100^{\text {th }}-$ the $300^{\text {th }}$ and the $500^{\text {th }}-$ the $1000^{\text {th }}$ iterations for the Bernoulli distribution. The difference between the Uniform and the Bernoulli distribution is as follows: $\mathrm{CW}, \mathrm{MD}$, and $\mathrm{MH}$ have a smaller minimal deviation at each iteration for the Uniform distribution. LD also achieves better results for the Uniform distribution except for the $100^{\text {th }}$ iteration. $\mathrm{BC}$ is paradoxically better for the Bernoulli distribution except for the $100^{\text {th }}$ iteration. BM have a smaller minimal deviation at each iteration for the Bernoulli distribution.

Comparing the results in the strongly and the weakly connected networks, we can see that the maximal and the minimal deviations are smaller at each iteration, for each distribution of the initial values, and for each examined model in the strongly connected networks.

From the results, we can also see an interesting phenomenon related to BC. In the strongly connected networks, it achieves the largest maximal precision at eight iterations for the Gaussian distribution and at nine iterations for the Uniform distribution. So, this weight model achieves the largest maximal precision at the most iterations in the strongly connected networks. Paradoxically, in the weakly connected networks, this weight achieves the largest minimal deviations at all the iterations when the initial states are of the Bernoulli distribution.

\section{CONCLUSION}

We examined the precision of the chosen weight models in various phases of the average consensus algorithm. We focused our attention on the Constant, the Maximum Degree, the Metropolis-Hastings, the Local Degree, the Best Constant and the Biphasically configured Metropolis-Hastings weight model. We examined the deviation of the least precise estimate from the real value of the average in ten strongly and ten weakly connected networks for the initial states of a Gaussian, a Uniform and a Bernoulli distribution. The first experiment was focused on finding the most and the least precise weight model for each iteration and for each distribution of the initial values. We can see that the Biphasically configured Metropolis-Hastings weight model achieved the highest precision in the most cases (in $80.83 \%$ ). The second most precise model is the Best Constant weight model with $17.17 \%$ of the cases. However, this weight model achieves also the lowest precision in $12.67 \%$ of the cases. In the strongly connected networks, this model achieved the highest precision more often in later phases of the algorithm, while, in the weakly connected ones, it achieved the highest precision in later phases. The third most precise model (and the last one that achieves the highest precision at least in one case) is the Local Degree weight model, which achieves the highest precision in $2 \%$ of the cases. Regarding the models with the lowest precision, the Constant weight model was the least precise in $87.33 \%$ cases. The second and also the last model that achieves the lowest precision at least once is the Best Constant weight model - as mentioned earlier, it achieved the lowest precision in $12.67 \%$ of all the cases. This model was especially imprecise for the Bernoulli distribution. 
The next experiment dealt with the average, the maximal and the minimal precision for each examined iteration and each distribution of the initial values. The results confirmed the previous experiment and so, a very high precision of the Biphasically configured Metropolis-Hastings weight model was proved (especially, for the Bernoulli distribution of the initial values). However, we can see the interesting phenomenon that the Best Constant weight model achieves the highest maximal precision at many examined iterations in the strongly connected networks.

APPENDIX

TABLE I

AVERAGE DEVIATION OF LEAST PRECISE ESTIMATES

\begin{tabular}{|c|c|c|c|c|c|c|c|c|c|c|c|}
\hline \multicolumn{12}{|c|}{ Average deviation of least precise estimates in strongly connected networks } \\
\hline \multirow[t]{2}{*}{$\mathrm{d}$} & $\mathrm{m}$ & \multicolumn{10}{|c|}{ Label of iteration } \\
\hline & & 100 it. & 200 it. & 300 it. & 400 it. & 500 it. & 600 it. & 700 it. & 800 it. & 900 it. & 1000 it. \\
\hline \multirow{6}{*}{ 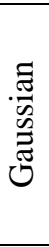 } & $\mathrm{CW}$ & $8,3 \mathrm{E}-01$ & $3,8 \mathrm{E}-01$ & $2,0 \mathrm{E}-01$ & $1,2 \mathrm{E}-01$ & 7,4E-02 & $4,8 \mathrm{E}-02$ & $3,2 \mathrm{E}-02$ & $2,2 \mathrm{E}-02$ & $1,6 \mathrm{E}-02$ & $1,1 \mathrm{E}-02$ \\
\hline & MD & 3,8E-01 & 1,2E-01 & $4,8 \mathrm{E}-02$ & 2,2E-02 & $1,1 \mathrm{E}-02$ & $5,5 \mathrm{E}-03$ & 2,9E-03 & $1,5 \mathrm{E}-03$ & 7,9E-04 & $4,1 \mathrm{E}-04$ \\
\hline & $\mathrm{MH}$ & $9,6 \mathrm{E}-02$ & $1,0 \mathrm{E}-02$ & $1,5 \mathrm{E}-03$ & $2,5 \mathrm{E}-04$ & 4,0E-05 & $6,8 \mathrm{E}-06$ & 1,2E-06 & 2,0E-07 & 3,3E-08 & 5,7E-09 \\
\hline & LD & $8,2 \mathrm{E}-02$ & 7,7E-03 & $9,6 \mathrm{E}-04$ & $1,3 \mathrm{E}-04$ & 1,9E-05 & $2,8 \mathrm{E}-06$ & 4,0E-07 & 5,8E-08 & $8,5 \mathrm{E}-09$ & 1,2E-09 \\
\hline & $\mathrm{BC}$ & $2,3 \mathrm{E}-01$ & $3,0 \mathrm{E}-02$ & $7,1 \mathrm{E}-03$ & $2,0 \mathrm{E}-03$ & $5,7 \mathrm{E}-04$ & $1,6 \mathrm{E}-04$ & $4,9 \mathrm{E}-05$ & $1,4 \mathrm{E}-05$ & $4,2 \mathrm{E}-06$ & $1,2 \mathrm{E}-06$ \\
\hline & $\mathrm{BM}$ & $7,3 \mathrm{E}-02$ & $5,5 \mathrm{E}-03$ & 5,3E-04 & $5,7 \mathrm{E}-05$ & $6,3 \mathrm{E}-06$ & $7,2 \mathrm{E}-07$ & $8,2 \mathrm{E}-08$ & $9,5 \mathrm{E}-09$ & $1,1 \mathrm{E}-09$ & $1,4 \mathrm{E}-10$ \\
\hline \multirow{6}{*}{$\stackrel{\Xi}{\Xi}$} & $\mathrm{CW}$ & $8,5 \mathrm{E}+01$ & $4,5 \mathrm{E}+01$ & $2,8 \mathrm{E}+01$ & $1,7 \mathrm{E}+01$ & $1,1 \mathrm{E}+01$ & $7,5 \mathrm{E}+00$ & $5,1 \mathrm{E}+00$ & $3,6 \mathrm{E}+00$ & $2,5 \mathrm{E}+00$ & $1,8 \mathrm{E}+00$ \\
\hline & MD & $4,5 \mathrm{E}+01$ & $1,7 \mathrm{E}+01$ & $7,5 \mathrm{E}+00$ & $3,6 \mathrm{E}+00$ & $1,8 \mathrm{E}+00$ & $9,1 \mathrm{E}-01$ & 4,7E-01 & $2,4 \mathrm{E}-01$ & 1,3E-01 & $6,8 \mathrm{E}-02$ \\
\hline & $\mathrm{MH}$ & $1,1 \mathrm{E}+01$ & $1,3 \mathrm{E}+00$ & $2,0 \mathrm{E}-01$ & $3,4 \mathrm{E}-02$ & $5,6 \mathrm{E}-03$ & $9,5 \mathrm{E}-04$ & $1,6 \mathrm{E}-04$ & 2,7E-05 & 4,6E-06 & 7,9E-07 \\
\hline & LD & $9,0 \mathrm{E}+00$ & $9,8 \mathrm{E}-01$ & $1,3 \mathrm{E}-01$ & 1,8E-02 & $2,6 \mathrm{E}-03$ & $3,8 \mathrm{E}-04$ & $5,5 \mathrm{E}-05$ & $8,0 \mathrm{E}-06$ & 1,2E-06 & $1,7 \mathrm{E}-07$ \\
\hline & $\mathrm{BC}$ & $3,1 \mathrm{E}+01$ & $6,2 \mathrm{E}+00$ & $1,7 \mathrm{E}+00$ & 4,7E-01 & 1,4E-01 & $4,0 \mathrm{E}-02$ & $1,2 \mathrm{E}-02$ & $3,5 \mathrm{E}-03$ & $1,0 \mathrm{E}-03$ & $3,0 \mathrm{E}-04$ \\
\hline & $\mathrm{BM}$ & $7,8 \mathrm{E}+00$ & $6,8 \mathrm{E}-01$ & $6,9 \mathrm{E}-02$ & 7,7E-03 & $8,6 \mathrm{E}-04$ & $9,8 \mathrm{E}-05$ & $1,1 \mathrm{E}-05$ & $1,3 \mathrm{E}-06$ & $1,5 \mathrm{E}-07$ & $1,7 \mathrm{E}-08$ \\
\hline \multirow{6}{*}{ 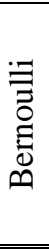 } & $\mathrm{CW}$ & $1,4 \mathrm{E}+02$ & $6,1 \mathrm{E}+01$ & $3,1 \mathrm{E}+01$ & $1,6 \mathrm{E}+01$ & $8,7 \mathrm{E}+00$ & $4,9 \mathrm{E}+00$ & $2,8 \mathrm{E}+00$ & $1,7 \mathrm{E}+00$ & $1,0 \mathrm{E}+00$ & $6,4 \mathrm{E}-01$ \\
\hline & MD & $6,1 \mathrm{E}+01$ & $1,6 \mathrm{E}+01$ & $4,9 \mathrm{E}+00$ & $1,7 \mathrm{E}+00$ & $6,3 \mathrm{E}-01$ & $2,6 \mathrm{E}-01$ & 1,2E-01 & 5,7E-02 & $2,8 \mathrm{E}-02$ & $1,5 \mathrm{E}-02$ \\
\hline & $\mathrm{MH}$ & $1,2 \mathrm{E}+01$ & 8,9E-01 & $9,8 \mathrm{E}-02$ & $1,4 \mathrm{E}-02$ & 2,3E-03 & $3,7 \mathrm{E}-04$ & $6,3 \mathrm{E}-05$ & $1,1 \mathrm{E}-05$ & 1,8E-06 & $3,1 \mathrm{E}-07$ \\
\hline & $\mathrm{LD}$ & $1,0 \mathrm{E}+01$ & $6,4 \mathrm{E}-01$ & $6,3 \mathrm{E}-02$ & $8,0 \mathrm{E}-03$ & $1,1 \mathrm{E}-03$ & $1,6 \mathrm{E}-04$ & $2,3 \mathrm{E}-05$ & 3,3E-06 & $4,8 \mathrm{E}-07$ & $7,1 \mathrm{E}-08$ \\
\hline & $\mathrm{BC}$ & $5,4 \mathrm{E}+01$ & $1,1 \mathrm{E}+01$ & $3,0 \mathrm{E}+00$ & $8,3 \mathrm{E}-01$ & $2,4 \mathrm{E}-01$ & $7,1 \mathrm{E}-02$ & $2,1 \mathrm{E}-02$ & $6,2 \mathrm{E}-03$ & $1,8 \mathrm{E}-03$ & $5,4 \mathrm{E}-04$ \\
\hline & $\mathrm{BM}$ & 3,7E-02 & $2,2 \mathrm{E}-03$ & $2,0 \mathrm{E}-04$ & $2,0 \mathrm{E}-05$ & 2,2E-06 & $2,4 \mathrm{E}-07$ & 2,7E-08 & $3,1 \mathrm{E}-09$ & $3,6 \mathrm{E}-10$ & $4,1 \mathrm{E}-11$ \\
\hline \multicolumn{12}{|c|}{ Average deviation of least precise estimates in weakly connected networks } \\
\hline \multirow{6}{*}{ 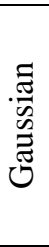 } & $\mathrm{CW}$ & $3,7 \mathrm{E}+00$ & $2,1 \mathrm{E}+00$ & $1,4 \mathrm{E}+00$ & $1,0 \mathrm{E}+00$ & $7,8 \mathrm{E}-01$ & $6,2 \mathrm{E}-01$ & $5,2 \mathrm{E}-01$ & $4,6 \mathrm{E}-01$ & 4,1 & 01 \\
\hline & MD & $2,1 \mathrm{E}+00$ & $1,0 \mathrm{E}+00$ & $6,2 \mathrm{E}-01$ & 4,6E-01 & 3,7E-01 & $3,0 \mathrm{E}-01$ & $2,5 \mathrm{E}-01$ & $2,1 \mathrm{E}-01$ & $1,8 \mathrm{E}-01$ & $1,6 \mathrm{E}-01$ \\
\hline & $\mathrm{MH}$ & $9,2 \mathrm{E}-01$ & $4,6 \mathrm{E}-01$ & $3,1 \mathrm{E}-01$ & 2,2E-01 & 1,6E-01 & $1,2 \mathrm{E}-01$ & $9,5 \mathrm{E}-02$ & $7,4 \mathrm{E}-02$ & $5,7 \mathrm{E}-02$ & $4,3 \mathrm{E}-02$ \\
\hline & $\mathrm{LD}$ & $8,1 \mathrm{E}-01$ & $4,0 \mathrm{E}-01$ & $2,6 \mathrm{E}-01$ & 1,8E-01 & 1,3E-01 & $9,6 \mathrm{E}-02$ & 7,2E-02 & $5,3 \mathrm{E}-02$ & 3,9E-02 & $2,9 \mathrm{E}-02$ \\
\hline & $\mathrm{BC}$ & $1,5 \mathrm{E}+00$ & 7,5E-01 & $5,1 \mathrm{E}-01$ & $3,5 \mathrm{E}-01$ & 2,6E-01 & 1,9E-01 & 1,4E-01 & 1,1E-01 & $8,4 \mathrm{E}-02$ & $6,5 \mathrm{E}-02$ \\
\hline & $\mathrm{BM}$ & 6,7E-01 & 3,7E-01 & $2,2 \mathrm{E}-01$ & $1,4 \mathrm{E}-01$ & $9,5 \mathrm{E}-02$ & $6,6 \mathrm{E}-02$ & 4,6E-02 & $3,3 \mathrm{E}-02$ & $2,4 \mathrm{E}-02$ & $1,7 \mathrm{E}-02$ \\
\hline \multirow{6}{*}{$\stackrel{\Xi}{\Xi}$} & $\mathrm{CW}$ & $1,6 \mathrm{E}+02$ & $1,0 \mathrm{E}+02$ & $8,4 \mathrm{E}+01$ & $6,9 \mathrm{E}+01$ & $5,7 \mathrm{E}+01$ & $4,9 \mathrm{E}+01$ & $4,2 \mathrm{E}+01$ & $3,6 \mathrm{E}+01$ & $3,2 \mathrm{E}+01$ & $2,8 \mathrm{E}+01$ \\
\hline & MD & $1,1 \mathrm{E}+02$ & $6,9 \mathrm{E}+01$ & $4,9 \mathrm{E}+01$ & $3,6 \mathrm{E}+01$ & $2,8 \mathrm{E}+01$ & $2,2 \mathrm{E}+01$ & $1,7 \mathrm{E}+01$ & $1,4 \mathrm{E}+01$ & $1,1 \mathrm{E}+01$ & $9,0 \mathrm{E}+00$ \\
\hline & $\mathrm{MH}$ & $6,2 \mathrm{E}+01$ & $3,2 \mathrm{E}+01$ & $1,9 \mathrm{E}+01$ & $1,3 \mathrm{E}+01$ & $8,8 \mathrm{E}+00$ & $6,4 \mathrm{E}+00$ & $4,7 \mathrm{E}+00$ & $3,4 \mathrm{E}+00$ & $2,6 \mathrm{E}+00$ & $2,0 \mathrm{E}+00$ \\
\hline & LD & $5,4 \mathrm{E}+01$ & $2,7 \mathrm{E}+01$ & $1,6 \mathrm{E}+01$ & $1,0 \mathrm{E}+01$ & $6,8 \mathrm{E}+00$ & $4,7 \mathrm{E}+00$ & $3,4 \mathrm{E}+00$ & $2,4 \mathrm{E}+00$ & $1,8 \mathrm{E}+00$ & $1,3 \mathrm{E}+00$ \\
\hline & $\mathrm{BC}$ & $1,3 \mathrm{E}+02$ & $8,4 \mathrm{E}+01$ & $5,5 \mathrm{E}+01$ & $3,8 \mathrm{E}+01$ & $2,7 \mathrm{E}+01$ & $2,0 \mathrm{E}+01$ & $1,5 \mathrm{E}+01$ & $1,2 \mathrm{E}+01$ & $9,0 \mathrm{E}+00$ & $6,9 \mathrm{E}+00$ \\
\hline & $\mathrm{BM}$ & $4,2 \mathrm{E}+01$ & $2,0 \mathrm{E}+01$ & $1,1 \mathrm{E}+01$ & $6,8 \mathrm{E}+00$ & $4,5 \mathrm{E}+00$ & $3,0 \mathrm{E}+00$ & $2,0 \mathrm{E}+00$ & $1,4 \mathrm{E}+00$ & $1,0 \mathrm{E}+00$ & $7,2 \mathrm{E}-01$ \\
\hline \multirow{6}{*}{ 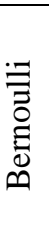 } & $\mathrm{CW}$ & $3,0 \mathrm{E}+02$ & $2,1 \mathrm{E}+02$ & $1,6 \mathrm{E}+02$ & $1,3 \mathrm{E}+02$ & $1,1 \mathrm{E}+02$ & $9,7 \mathrm{E}+01$ & $8,6 \mathrm{E}+01$ & $7,6 \mathrm{E}+01$ & $6,6 \mathrm{E}+01$ & $6,0 \mathrm{E}+01$ \\
\hline & MD & $2,1 \mathrm{E}+02$ & $1,3 \mathrm{E}+02$ & $9,8 \mathrm{E}+01$ & $7,6 \mathrm{E}+01$ & $6,0 \mathrm{E}+01$ & $4,8 \mathrm{E}+01$ & $3,9 \mathrm{E}+01$ & $3,2 \mathrm{E}+01$ & $2,7 \mathrm{E}+01$ & $2,2 \mathrm{E}+01$ \\
\hline & $\mathrm{MH}$ & $1,3 \mathrm{E}+02$ & $7,5 \mathrm{E}+01$ & $5,0 \mathrm{E}+01$ & $3,4 \mathrm{E}+01$ & $2,4 \mathrm{E}+01$ & $1,8 \mathrm{E}+01$ & $1,3 \mathrm{E}+01$ & $9,6 \mathrm{E}+00$ & $7,3 \mathrm{E}+00$ & $5,5 \mathrm{E}+00$ \\
\hline & LD & $1,1 \mathrm{E}+02$ & $6,5 \mathrm{E}+01$ & $4,2 \mathrm{E}+01$ & $2,8 \mathrm{E}+01$ & $1,9 \mathrm{E}+01$ & $1,3 \mathrm{E}+01$ & $9,5 \mathrm{E}+00$ & $6,8 \mathrm{E}+00$ & $5,0 \mathrm{E}+00$ & $3,6 \mathrm{E}+00$ \\
\hline & $\mathrm{BC}$ & $4,8 \mathrm{E}+02$ & $3,5 \mathrm{E}+02$ & $2,5 \mathrm{E}+02$ & $1,9 \mathrm{E}+02$ & $1,5 \mathrm{E}+02$ & $1,1 \mathrm{E}+02$ & $8,6 \mathrm{E}+01$ & $6,9 \mathrm{E}+01$ & $5,4 \mathrm{E}+01$ & $4,3 \mathrm{E}+01$ \\
\hline & $\mathrm{BM}$ & 3,9E-01 & $2,3 \mathrm{E}-01$ & $1,5 \mathrm{E}-01$ & $9,9 \mathrm{E}-02$ & $6,8 \mathrm{E}-02$ & 4,7E-02 & $3,3 \mathrm{E}-02$ & $2,3 \mathrm{E}-02$ & $1,6 \mathrm{E}-02$ & $1,2 \mathrm{E}-02$ \\
\hline
\end{tabular}


TABLE II

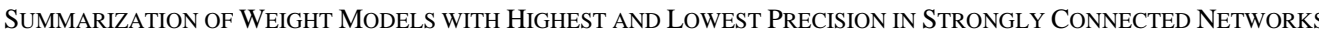

\begin{tabular}{|c|c|c|c|c|c|c|c|c|c|c|c|c|c|c|c|c|c|c|c|c|c|}
\hline \multicolumn{22}{|c|}{ Highest and lowest precision of least precise estimates in strongly connected networks } \\
\hline \multirow[t]{3}{*}{$\mathrm{d}$} & $\mathrm{m}$ & \multicolumn{20}{|c|}{ Label of iteration } \\
\hline & & \multicolumn{2}{|c|}{100 it. } & \multicolumn{2}{|c|}{200 it. } & \multicolumn{2}{|c|}{300 it. } & \multicolumn{2}{|c|}{400 it. } & \multicolumn{2}{|c|}{500 it. } & \multicolumn{2}{|c|}{600 it. } & \multicolumn{2}{|c|}{700} & \multicolumn{2}{|c|}{800 it. } & \multicolumn{2}{|c|}{900 it. } & 100 & \\
\hline & & $\mathrm{B}$ & $\mathrm{W}$ & $\mathrm{B}$ & $\mathrm{W}$ & $\mathrm{B}$ & $\mathrm{W}$ & B & $\mathrm{W}$ & $\mathrm{B}$ & $\mathrm{W}$ & $\mathrm{B}$ & $\mathrm{W}$ & $\mathrm{B}$ & $\mathrm{W}$ & & $\mathrm{W}$ & & $\mathrm{W}$ & B & $\mathrm{W}$ \\
\hline & $\mathrm{CW}$ & - & 10 & - & 10 & - & 10 & - & 10 & - & 10 & - & 10 & - & 10 & - & 10 & - & 10 & - & 10 \\
\hline$\Xi$ & MD & - & - & - & - & - & - & - & - & - & - & - & - & - & - & - & - & - & - & - & - \\
\hline$\cdot \vec{\sigma}$ & MH & - & - & - & - & - & - & - & - & - & - & - & - & - & - & - & - & - & - & - & - \\
\hline है & LD & - & - & - & - & - & - & - & - & - & - & - & - & - & - & - & - & - & - & - & - \\
\hline & $\mathrm{BC}$ & 3 & - & 3 & - & 4 & - & 5 & - & 5 & - & 5 & - & 5 & - & 5 & - & 6 & - & 6 & - \\
\hline & BM & 7 & - & 7 & - & 6 & - & 5 & - & 5 & - & 5 & - & 5 & - & 5 & - & 4 & - & 4 & - \\
\hline & CW & - & 10 & - & 10 & - & 10 & - & 10 & - & 10 & - & 10 & - & 10 & - & 10 & - & 10 & - & 10 \\
\hline$\exists$ & MD & - & - & - & - & - & - & - & - & - & - & - & - & - & - & - & - & - & - & - & - \\
\hline ह & MH & - & - & - & - & - & - & - & - & - & - & - & - & - & - & - & - & - & - & - & - \\
\hline 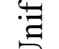 & LD & 2 & - & 1 & - & - & - & - & - & - & - & - & - & - & - & - & - & - & - & - & - \\
\hline & $\mathrm{BC}$ & - & - & 3 & - & 5 & - & 5 & - & 5 & - & 5 & - & 5 & - & 6 & - & 6 & - & 6 & - \\
\hline & BM & 8 & - & 6 & - & 5 & - & 5 & - & 5 & - & 5 & - & 5 & - & 4 & - & 4 & - & 4 & - \\
\hline & $\mathrm{CW}$ & - & 9 & - & 9 & - & 10 & - & 10 & - & 10 & - & 10 & - & 10 & - & 10 & - & 10 & - & 10 \\
\hline$\fallingdotseq$ & MD & - & - & - & - & - & - & - & - & - & - & - & - & - & - & - & - & - & - & - & - \\
\hline$\overline{\bar{\sigma}}$ & MH & - & - & - & - & - & - & - & - & - & - & - & - & - & - & - & - & - & - & - & - \\
\hline E & LD & - & - & - & - & - & - & - & - & - & - & - & - & - & - & - & - & - & - & - & - \\
\hline & $\mathrm{BC}$ & - & 1 & - & 1 & - & - & - & - & - & - & - & - & 2 & - & 2 & - & - & - & - & - \\
\hline & BM & 10 & - & 10 & - & 10 & - & 10 & - & 10 & - & 10 & - & 8 & - & 8 & - & 10 & - & 10 & - \\
\hline & CW & - & 29 & - & 29 & - & 30 & - & 30 & - & 30 & - & 30 & - & 30 & - & 30 & - & 30 & - & 30 \\
\hline & MD & - & - & - & - & - & - & - & - & - & - & - & - & - & - & - & - & - & - & - & - \\
\hline$\overline{\bar{\pi}}$ & MH & - & - & - & - & - & - & - & - & - & - & - & - & - & - & - & - & - & - & - & - \\
\hline$\stackrel{2}{2}$ & LD & 2 & - & 1 & - & - & - & - & - & - & - & - & - & - & - & - & - & - & - & - & - \\
\hline & $\mathrm{BC}$ & 3 & 1 & 6 & 1 & 9 & - & 10 & - & 10 & - & 10 & - & 12 & - & 13 & - & 12 & - & 12 & - \\
\hline & BM & 25 & - & 23 & - & 21 & - & 20 & - & 20 & - & 20 & - & 18 & - & 17 & - & 18 & - & 18 & - \\
\hline & & & ghes & and & lowe & $\mathrm{t}$ pre & cisio & of 1 & ast p & recis & esti & nate & $\overline{\text { in } \mathrm{W}}$ & eakly & cont & ectes & netv & orks & & & \\
\hline & CW & - & 10 & - & 10 & - & 10 & - & 10 & - & 10 & - & 10 & - & 10 & - & 10 & - & 10 & - & 10 \\
\hline & MD & - & - & - & - & - & - & - & - & - & - & - & - & - & - & - & - & - & - & - & - \\
\hline$\sqrt[\pi]{\infty}$ & MH & - & - & - & - & - & - & - & - & - & - & - & - & - & - & - & - & - & - & - & - \\
\hline సे & LD & - & - & - & - & - & - & - & - & - & - & - & - & - & - & - & - & - & - & - & - \\
\hline & $\mathrm{BC}$ & 2 & - & 1 & - & 1 & - & 1 & - & 1 & - & - & - & - & - & - & - & - & - & - & - \\
\hline & BM & 8 & - & 9 & - & 9 & - & 9 & - & 9 & - & 10 & - & 10 & - & 10 & - & 10 & - & 10 & - \\
\hline & CW & - & 6 & - & 5 & - & 6 & - & 6 & - & 6 & - & 7 & - & 7 & - & 9 & - & 9 & - & 9 \\
\hline & MD & - & - & - & - & - & - & - & - & - & - & - & - & - & - & - & - & - & - & - & - \\
\hline 0 & MH & - & - & - & - & - & - & - & - & - & - & - & - & - & - & - & - & - & - & - & - \\
\hline$\Xi$ & LD & 2 & - & - & - & 1 & - & - & - & 1 & - & 1 & - & 1 & - & 1 & - & 1 & - & 1 & - \\
\hline & $\mathrm{BC}$ & - & 4 & - & 5 & - & 4 & - & 4 & - & 4 & - & 3 & - & 3 & - & 1 & - & 1 & - & 1 \\
\hline & BM & 8 & - & 10 & - & 9 & - & 10 & - & 9 & - & 9 & - & 9 & - & 9 & - & 9 & - & 9 & - \\
\hline & CW & - & 3 & - & 5 & - & 6 & - & 6 & - & 6 & - & 6 & - & 6 & - & 6 & - & 6 & - & 6 \\
\hline & MD & - & - & - & - & - & - & - & - & - & - & - & - & - & - & - & - & - & - & - & - \\
\hline $\bar{\Xi}$ & MH & - & - & - & - & - & - & - & - & - & - & - & - & - & - & - & - & - & - & - & - \\
\hline$\exists$ & LD & - & - & - & - & - & - & - & - & - & - & - & - & - & - & - & - & - & - & - & - \\
\hline & $\mathrm{BC}$ & - & 7 & - & 5 & - & 4 & - & 4 & - & 4 & - & 4 & - & 4 & - & 4 & - & 4 & - & 4 \\
\hline & BM & 10 & - & 10 & - & 10 & - & 10 & - & 10 & - & 10 & - & 10 & - & 10 & - & 10 & - & 10 & - \\
\hline & $\mathrm{CW}$ & - & 19 & - & 20 & - & 22 & - & 22 & - & 22 & - & 23 & - & 23 & - & 25 & - & 25 & - & 25 \\
\hline & MD & - & - & - & - & - & - & - & - & - & - & - & - & - & - & - & - & - & - & - & - \\
\hline$\overline{\bar{\pi}}$ & MH & - & - & - & - & - & - & . & - & - & - & - & - & - & - & - & - & - & - & - & - \\
\hline$\stackrel{8}{2}$ & LD & 2 & - & - & - & 1 & - & - & - & 1 & - & 1 & - & 1 & - & 1 & - & 1 & - & 1 & - \\
\hline & $\mathrm{BC}$ & 2 & 11 & 1 & 10 & 1 & 8 & 1 & 8 & 1 & 8 & - & 7 & - & 7 & - & 5 & - & 5 & - & 5 \\
\hline & BM & 26 & - & 29 & - & 28 & - & 29 & - & 28 & - & 29 & - & 29 & - & 29 & - & 29 & - & 29 & - \\
\hline
\end{tabular}




\section{REFERENCES}

[1] F. Zhao and L. J. Guibas, Wireless Sensor Networks: An Information Processing Approach. Amsterdam, Netherlands: Morgan Kaufmann, 2004.

[2] L. Xiao, S. Boyd, and S. Lall, "A scheme for robust distributed sensor fusion based on average consensus," in Proc. of the 4th international symposium on Information processing in sensor networks, Los Angeles, CA, United States, 2005, pp. 63-70.

[3] A. Nayak and I. Stojmenovic, Wireless Sensor and Actuator Networks. Algorithms and Protocols for Scalable Coordination and Data Communication. Hoboken, New Jersey, USA: John Wiley \& Sons, 2010

[4] T. Zhao and A. Nehorai, "Distributed sequential Bayesian estimation of a diffusive source in wireless sensor networks," IEEE Trans. Signal Process., vol. 55, no. 4, pp. 1511-1524, March 2007. DOI 10.1109/TSP.2006.889975

[5] S. Barbarossa, S. Sardellitti, and P. Di Lorenzo, "Distributed detection and estimation in wireless sensor networks," in Academic Press Library in Signal Processing: Communications and Radar Signal Processing (Vol. 2),Waltham, MA, USA: Academic Press, 2014, pp. 329-408.

[6] V. Delouille, R. N. Neelamani and R. G. Baraniuk, "Robust distributed estimation using the embedded subgraphs algorithm," IEEE Trans. Signal Process., vol. 54, no. 8, pp. 2998-3010, Aug. 2006. DOI: 10.1109/TSP.2006.874839

[7] A. G. Dimakis, S. Kar, J. M. F. Moura, M. G. Rabbat, and A. Scaglione, "Gossip algorithms for distributed signal processing," Proc. IEEE, vol. 98, no. 11 , pp. 1847-1864, Nov. 2010. DOI: 10.1109/JPROC.2010.2052531

[8] G. Stamatescu, I. Stamatescu, and D. Popescu, "Consensus-based Data Aggregation for Wireless Sensor Networks," Journal of Control Engineering and Applied Informatics, vol. 19, no. 2, pp. 43-50, 2017.

[9] I. D. Schizas, A. Ribeiro, and G. B. Giannakis, "Consensus in ad hoc WSNs with noisy links - part I: Distributed estimation of deterministic signals," Trans. on Signal Process., vol. 56, no. 1, Jan. 2008. DOI: 10.1109/TSP.2007.906734

[10] M. Fanaei, M. C. Valenti, and N. A. Schmid, " Limited-feedback-based channel-aware power allocation for linear distributed estimation," in Proc. of the 47th Asilomar Conference on Signals, Systems and Computers, Pacific Grove, CA, United States, 2013, pp. 547-551. DOI 10.1109/ACSSC.2013.6810338

[11] M. Fanaei, "Distributed Detection and Estimation in Wireless Sensor Networks," Ph.D. dissertation, Ln. Dept. of Comp. Sci. Elect. Eng., West Virginia Univ., Morgantown, WV, USA, 2016.

[12] Y. R. Tsai and C. J. Chang, " Cooperative information aggregation for distributed estimation in wireless sensor networks," IEEE Trans. Signal Process., vol. 59, no. 8, pp. 3876-3888, Aug. 2011. DOI: 10.1109/TSP.2011.2153847

[13] A. Coluccia and G. Notarstefano, "A Bayesian Framework for Distributed Estimation of Arrival Rates in Asynchronous Networks," IEEE Trans. Signal Process., vol. 64, no. 15, pp. 3984-3996, Aug. 2016 DOI: $10.1109 /$ TSP.2016.2557313

[14] J. Li and G. AlRegib, "Distributed estimation in energy-constrained wireless sensor networks," IEEE Trans. Signal Process., vol. 57, no. 10 pp. 3746-3758, May 2009. DOI: 10.1109/TSP.2009.2022874

[15] O. Hlinka, O. Sluciak, F. Hlawastch, P.M. Djuric, and M. Rupp "Likelihood consensus and its application to distributed particle filtering," IEEE Trans. Signal Process., vol. 60, no. 8, pp. 4334-4349, Aug. 2012. DOI: 10.1109/TSP.2012.2196697

[16] A. Coluccia and G. Notarstefano: "Distributed estimation in uncalibrated heterogeneous networks", in Proc. of 20th World Congress of the International Federation of Automatic Control (IFAC), Toulouse, France), 2017

[17] G. S. Seyboth, D. V. Dimarogonas, and K. H. Johansson, “ Event-based broadcasting for multi-agent average consensus," Automatica, vol. 49, no. 1 , pp. 245-252, Jan. 2013. DOI: 10.1007/978-0-85729-033-5_3

[18] C. Nowzari and J. Cortés, "Zeno-free, distributed event-triggered communication and control for multi-agent average consensus," in Proc. of 2014 American Control Conference, ACC 2014, Portland, OR United States, 2014, pp. 2148-2153. DOI: 10.1109/ACC.2014.6859495

[19] P. Yang, R. A. Freeman, and K. M. Lynch, " Distributed cooperative active sensing using consensus filters," in Proc. of 2007 IEEE International Conference on Robotics and Automation, ICRA'07, Rome; Italy, 2007, pp. 405-410. DOI: 10.1109/ROBOT.2007.363820
[20] F. Woodcock and C. Engel, "Operational consensus forecasts," Weather and forecasting, vol. 20, no. 1, pp. 101-111, Feb. 2005. DOI 10.1175/WAF-831.1

[21] S. Vanka, V. Gupta, and M. Haenggi, "Power-delay analysis of consensus algorithms on wireless networks with interference," International Journal of Systems, Control and Communications, vol. 2 no. 1-3, pp. 256-274, 2010. DOI: 10.1504/IJSCC.2010.031166

[22] F. Garin and L. Schenato, "A survey on distributed estimation and contro applications using linear consensus algorithms," Lecture Notes in Control and Inform. Sci.., vol. 406, pp. 75-107, 2010. DOI: 10.1007/978-0-85729-033-5_3

[23] V. Schwarz and G. Matz, "Average consensus in wireless sensor networks: Will it blend?," in Proc. of 2013 IEEE International Conference on Acoustics, Speech and Signal Processing, Vancouver, BC, Canada, 2013, pp. 4584-4588. DOI 10.1109/ICASSP.2013.6638528

[24] G. Xiong and S. Kishore, "Linear high-order distributed average consensus algorithm in wireless sensor networks," EURASIP $J$ Adv Signal Process, vol. 1, no. 1, Dec. 2010, Art. no. 373604. DOI: $10.1155 / 2010 / 373604$

[25] D. Silvestre, P. Rosa, J. P. Hespanha, and C. Silvestre, "Finite-time average consensus in a Byzantine environment using set-valued observers," in Proc.of 2014 American Control Conference, Portland, OR, USA, 2014, pp. 3023-3028. DOI: 10.1109/ACC.2014.6859426

[26] K. M. Al-Smoul, T.A. Al-Rawashdeh, and A.A. Al-Dahoud, "An Improved Solar Low Energy Adaptive Clustering Hierarchy (ISLEACH) Technique," International Journal of Communication Networks and Information Security, vol. 8, no. 3, pp. 221-226, Dec. 2016.

[27] R. Kanan and O. Elhassan, "A combined batteryless radio and wifi indoor positioning for hospital nursing," Journal of Communications Software and Systems, vol. 12, no. 1, pp. 34-44, March 2016, Art. no. 179648 DOI: $10.24138 /$ jcomss.v12i1.89

[28] W. N. Anderson and T. D. Morley, "Eigenvalues of the Laplacian of a graph.” Linear and multilinear algebra, vol. 18, no. 2, May 2007. DOI: 10.1080/03081088508817681.

[29] D. A. Spielman, The Laplacian (Lecture 2). 6 pages. [Online] Cited $2017-$ $07-15$ Available http://www.cs.yale.edu/homes/spielman/561/2009/lect02-09.pdf

[30] L. Xiao, S. Boyd, and S. J. Kim, "Distributed average consensus with least-mean-square deviation," J Parallel Distrib Comput., vol. 67, no. 1, pp. 33-46, Jan. 2007. DOI: 10.1016/j.jpdc.2006.08.010

[31] V. Schwarz, G. Hannak, and G. Matz, "On the convergence of average consensus with generalized metropolis-hasting weights," in Proc. of 2014 IEEE International Conference on Acoustics, Speech and Signal Processing (ICASSP), Florence, Italy, 2014, pp. 5442-5446. DOI: 10.1109/ICASSP.2014.6854643

[32] M. Kenyeres, J. Kenyeres, and V. Skorpil, "The distributed convergence classifier using the finite difference," Radioengineering, vol. 25, no. 1, pp. 148-155, Apr. 2016. DOI: 10.13164/re.2016.0148

[33] J. Kenyeres, J. Kenyeres, M. Rupp, and P. Farkas, “ WSN implementation of the average consensus algorithm," in Proc. of 17th European Wireless Conference 2011, EW 2011, Vienna, Austria, 2011, pp. 1-8.

[34] W. Li and Y. Jia, "Consensus-based distributed multiple model UKF for jump Markov nonlinear systems," IEEE Trans. Autom. Control, vol. 57, no. 1, pp. 227-233, Jan. 2012. DOI: 10.1109/TAC.2011.2161838

[35] L. Xiao and S. Boyd, "Fast linear iterations for distributed averaging," Syst. Control Lett., vol. 53, no. 1, pp. 65-78, Sept. 2004. DOI: 10.1016/j.sysconle.2004.02.022

[36] M. Kenyeres, J. Kenyeres, V. Skorpil, and R. Burget, "Distributed aggregate function estimation by Biphasically configured MetropolisHasting weight model," Radioengineering, vol. 26, no. 2, pp. 479-495, June 2017. DOI: 10.13164/re.2017.0479 


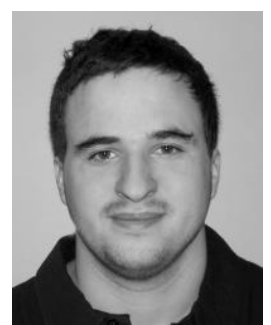

Martin Kenyeres (MSc) was born in Bratislava, Slovakia in 1988. He received his BSc. and MSc. from Slovak University Technology in Bratislava in 2011 and 2013, respectively. His research interests include distributed computing and wireless sensor networks. In 2013, he was with the Vienna University of Technology, Austria, where he participated in NFN SISE project under Professor Markus Rupp's supervision. He dealt with the implementation of distributed algorithms for an estimation of aggregate functions into wireless sensor networks. Since 2014, he has been with Brno University of Technology, where he works towards his $\mathrm{Ph}$.D. thesis on an analysis and an optimization of distributed systems.

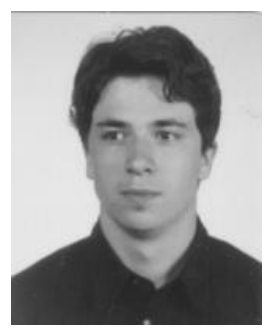

Jozef Kenyeres $(\mathrm{PhD})$ was born in Bratislava, Slovakia in 1985. He received his Ph.D. from the Slovak University of Technology in Bratislava in 2014. His research interests include embedded systems, wireless sensor networks, and VoIP. From 2006 to 2009, he worked as a technician at Slovak Telecom, a. s., Bratislava, Slovakia, From 2009 to 2013, he was a project assistant at Vienna University of Technology, Austria and from 2014 to 2015, he was with Zelisko, $\mathrm{GmbH}$, where he worked as a software developer. Since 2015, he has been working as a software developer at Sipwise GmbH, Austria. 